\title{
High Resolution Depth-Resolved Imaging From Multi-Focal Images for Medical
} Ultrasound

Diamantis, Konstantinos; Dalgarno, Paul A.; Greenaway, Alan H.; Anderson, Tom; Jensen, Jørgen Arendt; Sboros, Vassilis

\section{Published in:}

Proceedings of 37th Annual International Conference of the IEEE Engineering in Medicine and Biology Society (EMBS)

Link to article, DOI:

10.1109/EMBC.2015.7320020

Publication date:

2015

Document Version

Peer reviewed version

Link back to DTU Orbit

Citation $(A P A)$ :

Diamantis, K., Dalgarno, P. A., Greenaway, A. H., Anderson, T., Jensen, J. A., \& Sboros, V. (2015). High Resolution Depth-Resolved Imaging From Multi-Focal Images for Medical Ultrasound. In Proceedings of 37th Annual International Conference of the IEEE Engineering in Medicine and Biology Society (EMBS) (pp. 70677070). IEEE. https://doi.org/10.1109/EMBC.2015.7320020

\section{General rights}

Copyright and moral rights for the publications made accessible in the public portal are retained by the authors and/or other copyright owners and it is a condition of accessing publications that users recognise and abide by the legal requirements associated with these rights.

- Users may download and print one copy of any publication from the public portal for the purpose of private study or research.

- You may not further distribute the material or use it for any profit-making activity or commercial gain

- You may freely distribute the URL identifying the publication in the public portal 


\title{
High Resolution Depth-Resolved Imaging From Multi-Focal Images for Medical Ultrasound
}

\author{
Konstantinos Diamantis, Paul A. Dalgarno, Alan H. Greenaway, Tom Anderson, \\ Jørgen Arendt Jensen and Vassilis Sboros
}

\begin{abstract}
An ultrasound imaging technique providing subdiffraction limit axial resolution for point sources is proposed. It is based on simultaneously acquired multi-focal images of the same object, and on the image metric of sharpness. The sharpness is extracted by image data and presents higher values for in-focus images. The technique is derived from biological microscopy and is validated here with simulated ultrasound data. A linear array probe is used to scan a point scatterer phantom that moves in depth with a controlled step. From the beamformed responses of each scatterer position the image sharpness is assessed. Values from all positions plotted together form a curve that peaks at the receive focus, which is set during the beamforming. Selection of three different receive foci for each acquired dataset will result in the generation of three overlapping sharpness curves. A set of three calibration curves combined with the use of a maximum-likelihood algorithm is then able to estimate, with high precision, the depth location of any emitter fron each single image. Estimated values are compared with the ground truth demonstrating that an accuracy of $28.6 \mu \mathrm{m}(0.13 \lambda)$ is achieved for a $4 \mathrm{~mm}$ depth range.
\end{abstract}

\section{INTRODUCTION}

In ultrasound, the spatial resolution of current imaging systems is determined by the diffraction limit, depending on the wavelength, the aperture size, and the duration of transmitted pulses [1], [2]. Higher resolution may be achieved with the use of shorter pulses, higher frequencies, or smaller transducer elements, but at the expense of beam penetration depth. High penetration depth, in the order of tens of centimeters, is usually desired to acquire images of most tissue structures. However, if increased resolution could be achieved, there are several cases mainly concerning microvascular diseases [3], where medical ultrasound could provide additional benefits. Nowadays, reasearch towards this direction is often combined with contrast microbubble utilization. With the aid of contrast agents [4] and the $a$ priori knowledge of point source scatter, high resolution images of vascular structure have been obtained [5]-[7]. This was accomplished by applying effective aberration correction

This work is supported by Heriot-Watt University, and by grant 82-2012-4 from the Danish Advanced Technology Foundation.

K. Diamantis is with the Institute of Biological Chemistry, Biophysics and Bioengineering (IB3), Heriot-Watt (HW) University, Edinburgh, UK (corresponding author, e-mail: kd96@hw.ac.uk)

P. A. Dalgarno is with IB3, HW P.A.Dalgarno@hw.ac.uk

A. H. Greenaway was with IB3, HW (now retired)

T. Anderson is with the School of Clinical Sciences, Centre of Cardiovascular Science, University of Edinburgh, UK tom. anderson@ed.ac.uk

J. A. Jensen is with the Department of Electrical Engineering, Center for Fast Ultrasound Imaging, Technical University of Denmark, Kgs. Lyngby, Denmark jajeelektro.dtu.dk

V. Sboros is with IB3, HW V.Sboros@hw.ac.uk methods based on high accuracy position estimation of individual bubbles $(2-3 \mu \mathrm{m}$ diameter).

With those few exceptions, sub-diffraction imaging is still limited in medical ultrasound compared to optical microscopy [8], [9]. Methods like the Single Molecule Localization Microscopy [10] (SMLM) rely on an understanding of optical Point Spread Function (PSF) to achieve down to $\lambda / 10$ optical resolution. Previous work [11] has used the image sharpness metric to extend SMLM to depth dimension. Image sharpness is a pixel-based measure of image quality, widely used in the past in astronomy for correction of distorted images [12]. This tool has been translated in biological microscopy for the depth estimation of fluorescent particles [13], [14]. The method makes use of multiplane microscopy, for example with a quadratic diffraction grating [15] and a maximum likelihood algorithm. Combined with the sharpness, this approach, provides an average of approximately $14 \mathrm{~nm}$ depth resolution when applied to images of unresolved targets that are not background limited $(\lambda=532 \mathrm{~nm})$. In this paper, the technique is introduced in ultrasound imaging and its feasibility is investigated with Field II [16], [17] simulated ultrasound data.

\section{METhODS}

\section{A. Beamforming}

Any commercial transducer can be used for the transmission of ultrasound, which is performed through single plane waves. All the transducer elements are used as both the transmitting and receiving aperture. The unfocussed beams offer the advantage of covering the whole image region with only one emission, which allows for high frame rate and multiple acquisitions. After the transmission, the acquired data are stored and receive processing can be utilized to achieve multi-focal images. The transducer element signals can be beamformed offline in multiple ways. The common way to process the received responses is the Delay-AndSum (DAS) beamformer [18]. The signals are time-delayed, weighted, and summed to form the maximized beamformer output, $B(t)$, that for an array probe with $M$ active elements in receive can be extracted by:

$$
B(t)=\sum_{m=0}^{M-1} w_{m}(t) x_{m}\left(t-\tau_{m}\right)=\mathbf{w}(t)^{H} X(t),
$$

where $t$ is the time index, $\mathbf{w}(t)=\left[w_{0}(t), w_{1}(t), \ldots, w_{M-1}(t)\right]^{H}$ is the vector of the apodization weights, $X(t)=\left[x_{0}(t-\right.$ $\left.\left.\tau_{0}\right), x_{1}\left(t-\tau_{1}\right), \ldots, x_{M-1}\left(t-\tau_{M-1}\right)\right]^{H}$ is the array of the transducer element signals, and $\tau_{m}$ is the time delay applied to 
the $m$ th receiving element, based on its distance from the focus point. (1) can be implemented separately for any $\tau_{M}$, thus focus in receive. Three different receive foci will result in three different beamformer outputs that will subsequently produce different, spatially distinct images of the same object. A single point scatterer can now move accross the axial direction with a controlled step, and raw scatter data for each position can be captured. The process is repeated until the scatterer covers a distance of several millimeters.

\section{B. Receive Processing Algortihm}

Following similar strategy outlined in [13], for each position and for each of the three acquired images the normalized sharpness value is assessed. This metric shows the degree of aberration on an image or region of an image, but is dominated by defocus. Its main characteristic is that it reaches its maximum value at minimum aberration [12]. Image sharpness is usually calculated as the name implies from images pixel intensities. It is estimated by summing the squared values of each pixel and is then normalized by dividing by the squared sum of all pixel intensities. However, since it is possible to access the data from which an ultrasound image is formed, the calculation will be made from the received signals instead. The image intensity is generally proportional to the square of the signal amplitudes. Therefore, the sharpness function is redefined by:

$$
S=\sum_{k=1}^{q} A_{k}^{4} /\left(\sum_{k=1}^{q} A_{k}^{2}\right)^{2},
$$

where $S$ is the normalized sharpness, $q$ is the number of raw samples, and $A_{k}$ is the amplitude value of the $k^{\text {th }}$ sample. All sharpness values plotted together over the point total displacement will then create three sharpness curves $(S$ curves). One sharpness value from a single $S$-curve would correspond to two possible $z$-positions (Fig. 1). The objective of the algorithm is to provide a unique estimate of each point scatterer position. This estimate becomes unambiguous since each position is characterized by three distinct sharpness values. The accuracy of the estimation needs to be determined and extraction of an error is necessary, therefore the whole procedure is repeated a number of times. Data from all repetitive simulations are used for the estimation of the probability density function (PDF), $P\left(S_{j} \mid z\right)$, in other words, the probability that a particular normalized sharpness value, $S_{j}$, will be measured from the raw data of a point source located at depth $z$, where $j$ denotes the focus in receive. Since the sharpness calculations for each receive focus, do not depend on each other and with $z$ being known, the probability for the set of $N$ sharpness measurements for all receive foci when a particle is located at $z$ can be written as:

$$
L\left(S_{1}, S_{2}, \ldots, S_{N} \mid z\right)=\prod_{j=1}^{N} P\left(S_{j} \mid z\right),
$$

where $L$ is the likelihood for the set of sharpness measurements $S_{1}, S_{2}, \ldots, S_{N}$ and $N$ is the number of the different selected receive foci. The maximum likelihood estimator (MLE) of the particle depth, $z$, is the value of $z$ for which
$L$ is maximized given an actual dataset $S_{1}, S_{2}, \ldots, S_{N}$ and the calibration PDFs, $P\left(S_{j} \mid z\right)$. For the PDF a Gamma distribution has been selected as it fits best the Lorentzian shape of the $S$ curves as depicted in Fig. 1, and the behavior of the variance on the measured sharpness (Fig. 2), and is given by:

$$
P\left(S_{j} \mid z\right)=\frac{e^{\bar{S}_{j}^{2}} \bar{S}_{j}^{\alpha-1}(z) \beta^{-\alpha}}{\Gamma(\alpha)},
$$

where $\alpha=\bar{S}_{j}^{2}(z) / \bar{\sigma}_{j}^{2}, \beta=\bar{\sigma}_{j}^{2} / \bar{S}_{j}^{2}(z), \bar{S}_{j}(z)$ represents the interpolated Lorentzian fit of the mean $S$-curve, $\bar{\sigma}_{j}^{2}$ the interpolated Lorentzian fit of the variance, both extracted by the repetitive simulations, and $\Gamma$ is the Gamma function. The estimated depth position is finally compared with the actual depth that is already known since the movement of the point is determined by the simulation setup.

\section{Simulation Setup}

A phantom including a single point scatterer at a depth of $40 \mathrm{~mm}$, is created and used as a target to simulate the optical particles. The phantom is scanned by single plane wave emissions, made by a $7 \mathrm{MHz}, 192$ element, linear array simulated transducer with $\lambda$ spacing. The central transducer element is located above the point target. The speed of sound, $c$ is set to $1540 \mathrm{~m} / \mathrm{s}$ and all the parameters of the scan are given in Table I. All simulations are carried out with Field II [16], [17] software and Matlab scripts are utilized for the data post-processing.

TABLE I

Simulation SETUP PARAMETERS

\begin{tabular}{lc}
\hline \hline & Transducer \\
\hline Transducer type & Linear array \\
Transducer element pitch & $208 \mu \mathrm{m}$ \\
Transducer element kerf & $35 \mu \mathrm{m}$ \\
Transducer element height & $4.5 \mathrm{~mm}$ \\
Center frequency, $f_{0}$ & $7 \mathrm{MHz}$ \\
Bandwidth & $60 \%$ fractional \\
Speed of sound, $c$ & $1540 \mathrm{~m} / \mathrm{s}$ \\
Wavelength, $\lambda=c / f_{0}$ & $220 \mu \mathrm{m}$ \\
Excitation pulse & Two-cycle sinusoid at $f_{0}$ \\
\hline \hline & \\
\hline Transmit apodization & Plane Wave Emission \\
Receive apodization & Hanning \\
Receive focus depth & $39 / 40 / 41 \mathrm{~mm}$ \\
Number of transmitting elements & 192 \\
Number of receiving elements, $M$ & 192 \\
Number of emissions & 1 \\
\hline \hline & \\
\hline & Particle Movement \\
Lowest point & $(x, z)=(0,32.5) \mathrm{mm}$ \\
Total distance covered & $(x, z)=(0,47.5) \mathrm{mm}$ \\
$z$-step between succesive emissions & $15 \mathrm{~mm}(\mathrm{axially} \mathrm{only})$ \\
\hline \hline
\end{tabular}

Raw data from a single unfocussed emission are acquired from all 192 channels individually in receive. The data are stored and then a new phantom is created, with the point scatterer moved $100 \mu \mathrm{m}$ with direction away from the transducers surface until it reaches the depth of $47.5 \mathrm{~mm}$. The process is followed for the opposite direction as well, until 
the point scatterer reaches a depth of $32.5 \mathrm{~mm}$. Therefore, there are 151 acquisitions overall with the point target covering a distance of exactly $15 \mathrm{~mm}$. For each acquisition the data are beamformed with three different foci in receive. The central receive focus has been selected at a depth of $40 \mathrm{~mm}$, that is the target's initial position and then the two other values are at $-1 \mathrm{~mm}$ and $+1 \mathrm{~mm}$ of the starting depth.

\section{RESULTS}

Using the parameters in Table I a set of three normalized sharpness values is calculated from each of the 151 acquired datasets, leading to the generation of three sharpness curves. The whole simulation is repeated 5 times. Field II would produce identical results from repetitive simulations, and to introduce a level of uncertainty, $10 \mathrm{~dB}$ white Gaussian noise has been added to the raw data. This way it is possible to extract the mean $S$-curves and their variance that are requisites for the MLE analysis. Mean and variance are shown in Fig. 1 and 2 respectively together with their Lorentzian fits for the case of receive focus at $40 \mathrm{~mm}$. The mean $S$-curve presents a correlation coefficient of 0.99 with its Lorentzian fit, preserving the shape of the equivalent optical curves. The same value for the variance curve is siginificantly lower, at 0.78 .

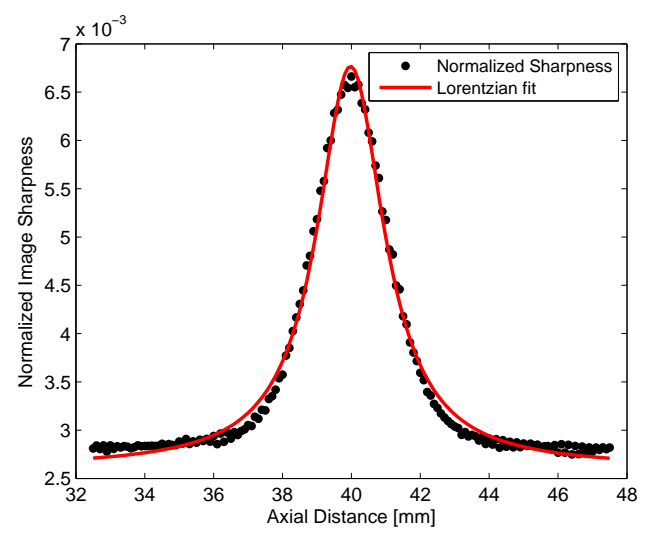

Fig. 1. Mean $S$-curve for a receive focus at $40 \mathrm{~mm}$ and its Lorentzian fit over point total displacement. The fit describes accurately the peak of the mean $S$-curve, which is crucial for the algorithm.

Both in optics and acoustics, sharpness metric changes in a similar manner when a single scatterer moves closer or away from focus. The sharpness variance demonstrates that with the addition of noise, Field II data from successive simulations are similar but not identical. In optics variance is higher around the $S$-curve peaks and lower away from them. This characteristic is preserved in ultrasonics as shown in Fig. 2. The Lorentzian fits of all $S$-curves and associated variance are then interpolated by a factor of 1000, using a Matlab spline interpolation function and are inserted to the selected PDF model. A set of three sharpness values as measured from one single acquisition are given as inputs to the algorithm, and the output is a prediction of depth position. This is the depth for which, the PDF presents its maximum value. Actual position of point target is known, as

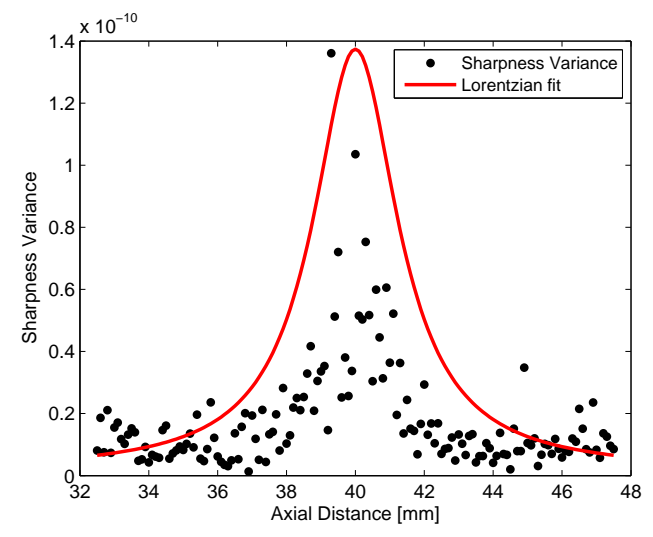

Fig. 2. Sharpness variance of the $S$-curve of Fig. 1 and its Lorentzian fit over point total displacement. The variance, although higher at the center, does not follow a particular pattern and the Lorentzian fit is only a basic approximation.

it is defined when creating the phantoms. Depth estimations for all 151 datasets, thus particle positions, are extracted and then compared with the ground truth. Since sharpness values are interpolated, the $z$-step is modulated accordingly and divided by 1000 , taking the value of $0.1 \mu \mathrm{m}$. The maximum of the PDF will correspond to a depth position of the updated depth vector using the new z-step. As a result, the error of the estimated depth will be assessed with a tenth of a micron accuracy.
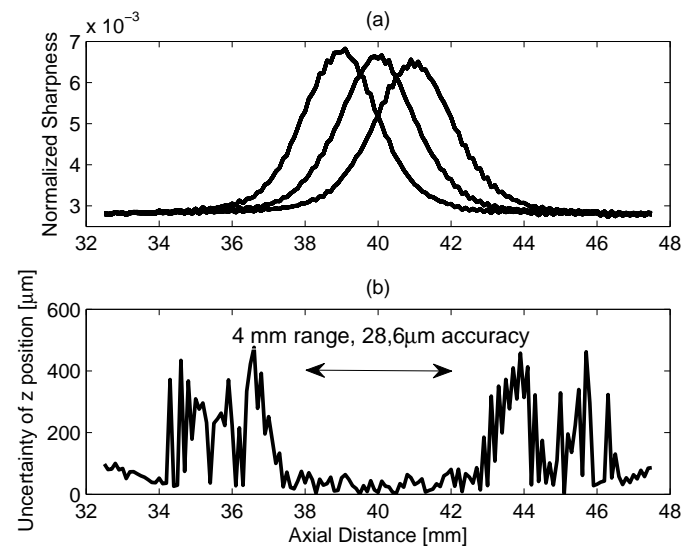

Fig. 3. Three sharpness curves (a) for Field II data of a point scatterer moving in depth. Each curve has been generated after beamforming the same data with a different focus in receive. A set of three sharpness values for each position of the point results in an estimation of the axial position with acuracy as shown in (b).

In Fig. 3 the three mean $S$-curves are plotted over axial distance and the absolute difference can also be found between estimated and real point position, which shows the accuracy of the proposed method. There is a central region of approximately $4 \mathrm{~mm}$ (from $38 \mathrm{~mm}$ to $42 \mathrm{~mm}$ ) where the localization error is on average, $28.6 \mu \mathrm{m}$ (see arrowed region in Fig. 3b), which almost corresponds to a value one order of magnitude lower than the used wavelength. The average accuracy for the total distance of $15 \mathrm{~mm}$ should not be considered, as the algorithm does not provide accurate estimates around both ends of the target's displacement. 
Fig. 3 and particularly the uncertainty bar presents good resemblance with the optics analogue that appears in [13]. In the optics case, the average achieved accuracy also becomes higher around the crossover points of the $S$-curves. However, it reaches a value almost 40 times lower than the used wavelength, indicating a further improvement compared to the ultrasonics equivalent.

\section{DISCUSSION}

A technique for improving the axial resolution of detection of point scatter using an ultrasound imaging method that is similar to those used clinically is suggested. Although, this is subject to further optimization, a localization error of an order of magnitude better than the wavelength was achieved, which is beyond the diffraction limit. The technique can be utilized directly with raw ultrasound data, and appears to be particularly suitable to the detection of single contrast microbubbles [5]-[7], which is a growing field of investigation.

In this example, the sharpness method performs best for a $4 \mathrm{~mm}$ region around the peak of the $S$-curves. This region is equal to 4 times the distance between two successive receive foci. At other parts of the $S$-curves there is no high difference between two neighbouring sharpness values, that is substantial for the method's high performance. Further research is needed to determine the optimal parameters, such as focal depths and sampling, that will help optimize the localization error, which appears further improved in the optics analogue. To increase the high-resolution range and possibly at the same time, reduce the uncertainty of the estimates it may be necessary to increase the number of overlapping $S$-curves to cover axially a whole image. A single image, or signals dataset will then be characterized by a set of several sharpness values. This is straightforward in ultrasound imaging as the receive data can be beamformed accordingly. Therefore the number of $S$-curves that can be formed is practically unlimited. Importantly, in multiplane microscopy this is more difficult to implement as light intensity per image plane drops. This fact is a significant advantage in the acoustics case, especially since the method is intended for real imaging.

On the other hand the Lorentzian fit may not be the optimal choice and the main source of error for the ultrasonics case. Unlike biological microscopy where the sharpness variance is calculated based on optical theory [13], the variance extracted from ultrasound data is only based on the addition of noise to the raw signals. The selection of the most appropriate fit needs to be reconsidered. All the above indicate that a calibration method needs to be generated in order to address the particle location. The validity of the technique needs to be evaluated with an experimental setup that matches the simulation in the first place, and next in real tissue environment where strong aberrations provide a variable and unpredictable point spread function.

\section{CONCLUSION}

Three or more simultaneous sharpness values can be extracted for each depth position of a moving target. Those values plotted together, form the sharpness curves from which a high accuracy estimate of all those positions can be extracted. This is the basis for the particle tracking technique in biological microscopy and analogous process can be reproduced for Field II simulated ultrasound data. The depth resolution achieved reaches $28.6 \mu \mathrm{m}(0.13 \lambda)$ for a $4 \mathrm{~mm}$ range of the total point displacement. The proposed method, translated from optics, presents encouraging results, but requires further investigation and experimental validation before being suitable for real-time applications.

\section{REFERENCES}

[1] P. Hoskins, K. Martin, and A. Thrush, "Diagnostic Ultrasound: Physics and Equipment," (2nd. ed.) Cambridge University Press, 2010.

[2] M. L. Oelze, and W. D. O’Brien, Jr., "Defining optimal axial and lateral resolution for estimating scatterer properties from volumes using ultrasound backscatter," J. Acoust. Soc. Am., vol. 115, no. 6, pp. 3226-3234, 2004.

[3] L. Pantoni, "Cerebral small vessel disease: From pathogenesis and clinical characteristics to therapeutic challenges," Lancet Neurol., vol. 9, no. 7, pp. 689-701, 2010.

[4] V. Sboros, C. M. Moran, S. D. Pye, and W. N. McDicken, "The behaviour of individual contrast agent microbubbles," Ultr. Med. Biol., vol. 29, no. 5, pp. 687-694, 2003.

[5] M. A. O'Reilly, R. M. Jones, and K. Hynynen, "Three-dimensional transcranial ultrasound imaging of microbubble clouds using a sparse hemispherical array," IEEE Trans. Biom. Eng., vol. 61, no. 4, pp. 12851294, 2014.

[6] M. A. O'Reilly, and K. Hynynen, "A super-resolution ultrasound method for brain vascular mapping," Med. Phys., vol. 40, no. 11, pp. 110701, 2013.

[7] K. Christensen-Jeffries, R. J. Browning, M. X. Tang, C. Dunsby, and R. J. Eckersley, "In Vivo Acoustic Super-Resolution and Super-Resolved Velocity Mapping Using Microbubbles," IEEE Trans. Med. Imag., vol. 34, no. 2, pp. 433-440, February 2015.

[8] M. J. Rust, M. Bates, and X. Zhuang, "Sub-diffraction-limit imaging by stohastic optical reconstruction microscopy (STORM)," Nature Methods, vol. 3, no. 10, pp. 793-796, 2006.

[9] E. Betzig, G. H. Patterson, R. Sougrat, O. W. Lindwasser, S. Olenych, J. S. Bonifacino, M. W. Davidson, J. Lippincott-Schwartz, and H. F. Hess, "Imaging intracellular fluorescent proteins at nanometer resolution," Science, vol. 313, no. 5793, pp. 1642-1645, 2006.

[10] A. L. McEvoy, D. Greenfield, M. Bates, J. Liphardt, "Q\&A: Singlemolecule localization microscopy for biological imaging," BMC Biology, vol. 8, no. 106, 2010.

[11] Y. Sun, J. D. McKenna, J. M. Murray, E. M. Ostap, and Y. E. Goldman, "Parallax: high accuracy three-dimensional single molecule tracking using split images," Nano Lett., vol. 9, no. 7, pp. 2676?2682, 2009.

[12] R. A. Muller, and A. Buffington, "Real-time correction of atmospherically degraded telescope images through image sharpnening," J. Opt. Soc. Am, vol. 64, no. 9, pp. 1200-1210, September 1974.

[13] H. I. C. Dalgarno, P. A. Dalgarno, A. C. Dada, C. E. Towers, G. J. Gibson, R. M. Parton, I. Davis, R. J. Warburton, and A. H. Greenaway, "Nanometric depth resolution from multi-focal images in microscopy," J. R. Soc. Interface, vol. 8, no. 60, pp. 942-951, July 2011.

[14] P. A. Dalgarno, H. I. C. Dalgarno, A. Putoud, R. Lambert, L. Paterson, D. C. Logan, D. P. Towers, R. J. Warbuton, and A. H. Greenaway, "Multiplane imaging and three dimensional nanoscale particle tracking in biological microscopy," Opt. Expr., vol. 18, no. 2, pp. 877-884, 2010.

[15] P. M. Blanchard, and A. H. Greenaway, "Simultaneous multiplane imaging with a distorted diffraction grating," Appl. Opt., vol. 38, no. 32, pp. 6692-6699, 1999.

[16] J. A. Jensen, "Field: A Program for Simulating Ultrasound Systems," Med. Biol. Eng. Comp., vol. 10th Nordic-Baltic Conference on Biomedical Imaging, Vol. 4, Supplement 1, Part 1, pp. 351-353, 1996.

[17] J. A. Jensen and N. B. Svendsen, "Calculation of pressure fields from arbitrarily shaped, apodized, and excited ultrasound transducers," IEEE Trans. Ultrason., Ferroelec., Freq. Contr., vol. 39, pp. 262-267, 1992.

[18] K. E. Thomenius, "Evolution of ultrasound beamformers," Proc. IEEE Ultrason. Symp., pp. 1615-1622, 1996. 